\title{
BMJ Open Effect of aspirin in takotsubo syndrome: protocol of a systematic review and meta-analysis
}

\author{
Jinhai Lin (D) , ${ }^{1}$ Bingxin Wu, ${ }^{1}$ Luoqi Lin, ${ }^{1}$ Yining Ding, ${ }^{1}$ Biying Zhong (D),${ }^{1}$ \\ Zhiwei Huang, ${ }^{1}$ Miaoyang Lin, ${ }^{1}$ Dan-Ping Xu (D) ${ }^{2}$
}

To cite: Lin J, Wu B, Lin L, et al. Effect of aspirin in takotsubo syndrome: protocol of a systematic review and meta-analysis. BMJ Open 2021;11:e046727. doi:10.1136/ bmjopen-2020-046727

- Prepublication history and additional supplemental material for this paper are available online. To view these files, please visit the journal online. (http://dx.doi.org/10.1136/ bmjopen-2020-046727)

Received 07 November 2020 Accepted 28 July 2021

\section{Check for updates}

\section{(c) Author(s) (or their} employer(s)) 2021. Re-use permitted under CC BY-NC. No commercial re-use. See rights and permissions. Published by BMJ.

${ }^{1}$ The Second Clinical Medical College, Guangzhou University of Chinese Medicine, Guangzhou, China

${ }^{2}$ Department of Traditional Chinese Medicine, The Eighth Affiliated Hospital of Sun Yat-sen University, Shenzhen, China

Correspondence to

Dr Dan-Ping Xu

xudanping@hotmail.com

\section{ABSTRACT}

Introduction Takotsubo syndrome (TTS) is a sudden reversible weakening of the left ventricle function induced by severe stress and resembles many features as acute coronary syndrome. Even though many guidelines had been published about TTS, there is no consensus regarding the long-term treatment. Aspirin is one of the most common prescribed medicines at discharge for patients with the intention to reduce thrombus events and improve the overall prognosis. However, existing studies yielded conflicting results concerning its effects. This study aims to evaluate the impact of long-term maintenance treatment of aspirin in TTS and provides insights in clinical management.

Methods and analysis After searching through electronic databases (PubMed, Embase, Cochrane Library, Web of Science, National Library of Medicine Gateway, CNKI, Wanfang and VIP), grey literatures, conference abstract and trial registries for clinical studies investigating the impact of aspirin on patients with TTS, a systemic review and meta-analysis will be conducted. The search will be limited from inception of each database to 1 August 2020. The outcomes including all-cause death, TTS recurrence, stroke, transient ischaemic attack or myocardial infarction at 30-day and 5-year follow-up will be examined. Risk of bias will be assessed by Newcastle-0ttawa quality assessment scale for observational studies and Cochrane Effective Practice and Organization of Care evaluation tool for interventional studies. Grading of Recommendations Assessment, Development and Evaluations method will be applied to assess the quality of evidence. If available, the effects of aspirin on the above outcomes for patients with TTS will be evaluated using random-effect modelling with relative risk at 95\% Cls. Subgroup analysis and sensitivity analysis will also be performed when possible.

Ethics and dissemination Ethics approval was not required due to the retrospective nature of the study. Results of the review will be published in a peer-reviewed journal.

PROSPERO registration number CRD42020212729.

\section{INTRODUCTION}

Takotsubo syndrome (TTS) refers to a reversible form of acute heart failure induced by severe stress which shares many characteristics with acute coronary syndrome (ACS), including demographic features, onset

\section{Strengths and limitations of this study}

- Eligible literature from existing common databases will be included in this study.

- The systematic review will be performed according to the Meta-analysis of Observational Studies in Epidemiology guideline and will be reported according to the Preferred Reporting Items for Systematic Reviews and Meta-Analysis guidelines.

- Subgroup analysis and sensitivity analysis will be performed to evaluate the bias and stability in the study.

- Given the lack of understanding on takotsubo syndrome, the number of finally included studies might be limited.

symptoms, ECG abnormalities and elevation of cardiac biomarkers. ${ }^{1}$ Due to a lack of noninvasive examinations method, the diagnosis and understanding of TTS remains poor. In clinical practice, TTS is mainly diagnosed by presentation of severe transient left ventricular (LV) dysfunction with typical apical ballooning or other regional wall motion abnormalities documented by coronary angiography with left ventriculography. ${ }^{2}$

Another characteristic feature of TTS is the reversible pathology change and self-limiting clinical course. ${ }^{3}$ For a rather long-time, TTS was generally considered as a benign disease with relatively low morbidity. ${ }^{4}$ However, this view is challenged by recent studies. ${ }^{56}$ Based on data obtained from the International Takotsubo Registry, ${ }^{7}$ the rate of major adverse cardiac and cerebrovascular events (MACCE) for patients with TTS was $9.9 \%$ per patientyear and the mortality was $5.6 \%$ per patientyear in long-term follow-up. ${ }^{8}$ In addition, the incidences of in-hospital complications including shock and death were also comparable to ACS. ${ }^{910}$ But the understanding and management of TTS is behind compared with ACS. Even though many guidelines had been 
published about TTS, there is no consensus regarding the long-term treatment. ${ }^{11}$

The LV thrombus and systematic embolism were reported to occur on approximately $2 \%-8 \%$ of all patients with TTS with LV abnormalities. ${ }^{12}$ In comparison, the incidence of thrombosis in TTS in 5-year follow-up was twofold higher than ACS (21\% vs 9\%), ${ }^{13}$ which indicated the necessity of applying antithrombotic therapy. ${ }^{14}$

Recent studies suggested that the release of catecholamines during acute phase of TTS initiates platelet aggregation, secretion and activation of arachidonate pathway. ${ }^{2}{ }^{15} 16$ Therefore, aspirin became one of the most commonly prescribed medicines at discharge for patients with TTS. ${ }^{17}$ However, it is important to acknowledge that existing studies yielded conflicting results concerning its effects. ${ }^{18-20}$ Prior systematic review and meta-analysis demonstrated that aspirin was unable to improve the prognosis of TTS. ${ }^{21}$ However, its conclusion was challenged by subsequent studies suggesting that more studies are needed to resolve this question. ${ }^{22}$

To evaluate the effect as well as safety of aspirin for patients with TTS, the protocol aims to systemically review existing literature and conduct a comprehensive meta-analysis to provide the most updated evidence for the treatment of TTS in clinical practice.

\section{METHODS AND ANALYSIS}

\section{Patient and public involvement}

There will be no patient or public involvement given the nature of the study.

\section{Study design}

The design of this systematic review was carried out according to the recommendation of the Preferred Reporting Items for Systematic Reviews and Meta-Analysis Protocols (PRISMA-P) statement. ${ }^{23}$ The protocol has also acquired approval automatically from the PROSPERO.

The systematic review will be performed according to the Meta-analysis of Observational Studies in Epidemiology guidelines and will be reported according to the PRISMA guidelines. ${ }^{24} 25$

\section{Search strategy}

The information to be included in this study will cover electronic databases, grey literatures, conference abstract and trial registries.

Databases including PubMed, Excerpta Medica Database (Embase), Cochrane Library, Web of Science, ClinicalTrials.gov, National Library of Medicine (NLM) Gateway, China National Knowledge Infrastructure (CNKI), Wanfang and Weipu (VIP) will be searched from inception of the library to 1 August 2020. The search will follow the principle of PICOS (participants, intervention, comparison, outcome and study design), employing subject words and free words for the term 'Takotsubo Cardiomyopathy' and 'Aspirin' with adjustment as necessary per specific database (see online supplemental file
1). Reference list of included studies will also be scanned for additional relevant articles.

The search is anticipated to be completed by November 2020.

\section{Eligibility criteria}

Participants and intervention

Patients with a diagnosis of TTS, regardless of the diagnostic criteria, will be included in the study. To estimate the long-term effect of aspirin, only studies in which participants taking aspirin for no less than 90 days will be included.

\section{Comparison}

The outcome measures for patients with TTS taking and not taking aspirin will be compared in the study.

\section{Study outcomes}

The co-primary outcome will be the incidence of MACCE, that is, a composite of all-cause death, TTS recurrence, stroke or transient ischaemic attack (TIA) or myocardial infarction (MI) at 30-day and 5-year follow-up.

The secondary outcome will be each component of MACCE. Included studies must contain at least one of the above outcome measurements.

Types of studies

Both observational and interventional studies are eligible for the study. Articles that published in languages other than English or Chinese will be translated into English when available.

\section{Exclusion}

Studies including participants with malignancies or autoimmune diseases or taking antithrombotic therapy for other diseases will be excluded. Review articles, commentaries, laboratory science studies, case studies, case series and other studies that do not meet the requirements aforementioned will be excluded.

\section{Study records}

Data management

Publication screening will be managed through EndNote software (V.X9, Clarivate Analytics), in which duplicates will be removed and further review will be performed.

\section{Selection process}

Two investigators will independently screen each record by the title and abstract before categorising all literatures into three groups: relevant, irrelevant and uncertain. After preliminary screening, irrelevant records agreed by both investigators will be eliminated, while the others will be retrieved by full text for further assessment. In the process, a list of eligible articles will be made separately by each reviewer. Discrepancies between the two lists will be resolved by discussion if possible. When an unsettled disagreement arises, a final decision will be made by consulting a third reviewer. Reference list of the 
included articles will also be reviewed to check for additional studies that might be omitted previously.

A flow diagram for the selection process will be drawn in accordance with the PRISMA guidelines.

\section{Data collection process}

Data extraction will be completed by one reviewer using a predesigned form.

Data to be collected will include study characteristics such as author, publication year, time period of study, country and site; cohort characteristics such as sample size, demographics (age, gender), diagnostic criteria, comorbid history and concomitant medication use; patient outcomes such as the incidence of all-cause death, TTS recurrence, stroke or TIA or MI at 30-day and 5-year follow-up.

Missing information will be estimated from the available data as appropriate. If the data are incomplete or unclear, we will contact the corresponding author. The results of extraction will be verified by two reviewers separately before data analysis and disagreements will be resolved from consultation from a third reviewer.

The process is expected to be completed by December 2020.

\section{Data synthesis}

The essential descriptions of all enrolled studies including type of publication, author, publication year and country will be included in the systematic review. A brief summary of the study design, participant characteristics, diagnostic criteria, sample size, follow-up and outcome measurements will be included for all studies. Subgroups analyses based on age, gender, region, commodity diseases, medication and each component of the outcomes will be measured if possible.

\section{Quality assessment and risk of bias}

Risk of bias will be assessed by independent researchers following the Newcastle-Ottawa quality assessment scale for observational studies and Cochrane Effective Practice and Organization of Care tool for interventional studies. ${ }^{26}$ Discrepancies will be resolved through discussion. A third reviewer will arbitrate unsettled disagreements. The Grading of Recommendations Assessment, Development and Evaluations will be applied to assess the quality of evidence for disease outcomes.

\section{Meta-analysis}

Meta-analysis will be performed using R software (V.4.0.2), in which package 'meta' (V.4.14-0) and package 'robvis' (V.0.3.0) will be used in the study.

Meta-analysis will be performed with different homogeneities among populations, which will be tested by the $\mathrm{I}^{2}$ statistics. $\mathrm{I}^{2}$ values of $0 \%-30 \%$ will be considered as minimal heterogeneity, 31\%-50\% moderate heterogeneity and $>50 \%$ substantial heterogeneity.

If available, the effects of aspirin on MACCE for patients with TTS will be evaluated using random-effect modelling. Relative risk with $95 \%$ CIs will be calculated for dichotomous data and weighted mean difference for continuous data.

Begg's funnel plot and Egger's linear regression will be used to assess the publication bias and sample size bias. An asymmetrical funnel plot or $p$ value of $<0.10$ on Egger's test will be considered as the presence of publication bias.

Subgroup analysis will be performed based on gender, country, diagnostic criteria, comorbid history, concomitant medication use and each component of MACCE if possible. Meta regression analysis will be used to explore potential source of heterogeneity if more than 10 studies were included in each measurement.

Sensitivity will be analysed by dropping one study at a time and measure stability of the analysis. Graphics of pooled data (forest plots, L'Abbé plot, radial plot and meta regression) will be plotted and added into the manuscript.

If pooled data remain heterogeneous within these pooled groups, a narrative description will be provided.

Contributors $\mathrm{JL}$ and D-PX were involved in conception and generation of the study protocol. JL, D-PX, BW, YD and BZ were involved in study design. $\mathrm{LL}$ and $\mathrm{ZH}$ will review the literatures for inclusion. BW will be responsible for resolving disagreements in data selection, synthesis and assessment. ML will verify the data. The manuscript is approved by all authors for publication.

Funding The authors have not declared a specific grant for this research from any funding agency in the public, commercial or not-for-profit sectors.

Competing interests None declared.

Patient consent for publication Not required.

Provenance and peer review Not commissioned; externally peer reviewed.

Supplemental material This content has been supplied by the author(s). It has not been vetted by BMJ Publishing Group Limited (BMJ) and may not have been peer-reviewed. Any opinions or recommendations discussed are solely those of the author(s) and are not endorsed by BMJ. BMJ disclaims all liability and responsibility arising from any reliance placed on the content. Where the content includes any translated material, BMJ does not warrant the accuracy and reliability of the translations (including but not limited to local regulations, clinical guidelines, terminology, drug names and drug dosages), and is not responsible for any error and/or omissions arising from translation and adaptation or otherwise.

Open access This is an open access article distributed in accordance with the Creative Commons Attribution Non Commercial (CC BY-NC 4.0) license, which permits others to distribute, remix, adapt, build upon this work non-commercially, and license their derivative works on different terms, provided the original work is properly cited, appropriate credit is given, any changes made indicated, and the use is non-commercial. See: http://creativecommons.org/licenses/by-nc/4.0/.

\section{ORCID iDs}

Jinhai Lin http://orcid.org/0000-0003-0745-0904

Biying Zhong http://orcid.org/0000-0001-5307-1695

Dan-Ping Xu http://orcid.org/0000-0002-4900-0699

\section{REFERENCES}

1 Ghadri J-R, Wittstein IS, Prasad A, et al. International expert consensus document on takotsubo syndrome (Part I): clinical characteristics, diagnostic criteria, and pathophysiology. Eur Heart $J$ 2018;39:2032-46.

2 Pelliccia F, Kaski JC, Crea F, et al. Pathophysiology of takotsubo syndrome. Circulation 2017;135:2426-41.

3 Akashi YJ, Goldstein DS, Barbaro G, et al. Takotsubo cardiomyopathy: a new form of acute, reversible heart failure. Circulation 2008;118:2754-62.

4 Templin C, Napp LC, Ghadri JR. Underestimated but understood? J Am Coll Cardiol 2016;67:1937-40. 
5 Dastidar AG, Frontera A, Palazzuoli A, et al. Takotsubo cardiomyopathy: unravelling the malignant consequences of a benign disease with cardiac magnetic resonance. Heart Fail Rev 2015;20:415-21.

6 Deshmukh A, Kumar G, Pant S, et al. Prevalence of takotsubo cardiomyopathy in the United States. Am Heart J 2012;164:66-71.

7 Templin C, Ghadri JR, Diekmann J, et al. Clinical features and outcomes of takotsubo (stress) cardiomyopathy. N Engl J Med 2015;373:929-38.

8 Boland TA, Lee VH, Bleck TP. Stress-Induced cardiomyopathy. Crit Care Med 2015;43:686-93.

9 Tornvall P, Collste O, Ehrenborg E, et al. A case-control study of risk markers and mortality in takotsubo stress cardiomyopathy. J Am Coll Cardiol 2016;67:1931-6.

10 Redfors B, Vedad R, Angerås O, et al. Mortality in takotsubo syndrome is similar to mortality in myocardial infarction - A report from the SWEDEHEART registry. Int J Cardiol 2015;185:282-9.

11 Medina de Chazal H, Del Buono MG, Keyser-Marcus L, et al. Stress Cardiomyopathy Diagnosis and Treatment: JACC State-of-the-Art Review. J Am Coll Cardiol 2018;72:1955-71.

12 Ghadri J-R, Wittstein IS, Prasad A, et al. International expert consensus document on takotsubo syndrome (Part II): diagnostic workup, outcome, and management. Eur Heart J 2018;39:2047-62.

13 El-Battrawy I, Gietzen T, Lang S, et al. Short- and long-term incidence of thromboembolic events in takotsubo syndrome as compared with acute coronary syndrome. Angiology 2019;70:838-43.

14 Dias A, Franco E, Koshkelashvili N, et al. Antiplatelet therapy in takotsubo cardiomyopathy: does it improve cardiovascular outcomes during index event? Heart Vessels 2016;31:1285-90.

15 Pirzer R, Elmas E, Haghi D, et al. Platelet and monocyte activity markers and mediators of inflammation in takotsubo cardiomyopathy. Heart Vessels 2012;27:186-92.
16 Anfossi G, Trovati M. Role of catecholamines in platelet function: pathophysiological and clinical significance. Eur J Clin Invest 1996;26:353-70.

17 Collado Moreno CC, Garcia-Gonzalez RF, Valiente-Aleman I, et al. Management of patients diagnosed with tako-tsubo syndrome. European Journal of Heart Failure 2018;20:487.

18 Bertaina M, D'Ascenzo F, lannaccone M, et al. 3113ls aspirin needed after takotsubo syndrome?: a propensity score sub-analysis of intertak registry. Eur Heart J 2017;38:647.

19 Fazio G, Pizzuto C, Barbaro G, et al. Chronic pharmacological treatment in takotsubo cardiomyopathy. Int $J$ Cardiol 2008:127:121-3.

20 Dias AM, Ross T, De Guevara EFL, et al. Does guideline directed medical therapy for systolic heart failure help prevent takotsubo recurrence and/or READMSSION? J Am Coll Cardiol 2020;75:879.

21 Santoro F, leva R, Musaico F, et al. Lack of efficacy of drug therapy in preventing takotsubo cardiomyopathy recurrence: a meta-analysis. Clin Cardiol 2014;37:434-9.

22 Abanador-Kamper N, Kamper L, Wolfertz J, et al. Evaluation of therapy management and outcome in takotsubo syndrome. BMC Cardiovasc Disord 2017;17:225.

23 Preferred reporting items for systematic review and meta-analysis protocols (PRISMA-P) 2015: elaboration and explanation. BMJ 2016;354:i4086.

24 Liberati A, Altman DG, Tetzlaff J, et al. The PRISMA statement for reporting systematic reviews and meta-analyses of studies that evaluate healthcare interventions: explanation and elaboration. $B M J$ 2009;339:b2700.

25 Stroup DF, Berlin JA, Morton SC, et al. Meta-Analysis of observational studies in epidemiology: a proposal for reporting. meta-analysis of observational studies in epidemiology (moose) group. JAMA 2000;283:2008-12.

26 Stang A. Critical evaluation of the Newcastle-Ottawa scale for the assessment of the quality of nonrandomized studies in metaanalyses. Eur J Epidemiol 2010;25:603-5. 